\title{
Quality of Experience aware multimedia provisioning with Cross-layer Network Management
}

\author{
René Serral-Gracià, Eva Marin-Tordera, Marcelo Yannuzzi, Xavi Masip-Bruin, Sergi Sánchez \\ Advanced Network Architectures Lab, Technical University of Catalunya (UPC), Spain \\ \{rserral, eva, yannuzzi, xmasip, sergio\}@ac.upc.edu
}

\begin{abstract}
This paper presents a Cross-layer Network Management System (NMS) that allows Service Providers (SPs) to perform cost-effective network resource reservations with their Network Operators (NOs). The novelty of our NMS is that it offers a fresh and promising approach to use the endusers satisfaction level as the metric to perform the resource management. We show that our system is capable of achieving considerable reductions in the operational costs of SPs, while keeping proper bounds in the end-user satisfaction for the offered multimedia services.
\end{abstract}

\section{INTRODUCTION}

Over years, Network Operators (NOs) have heavily relied in overprovisioning in order to provide network services to their customers. However, Internet traffic doubles every two years, so there are many arguments against overprovisioning, which seems unsustainable both in terms of operational and power consumption costs. Additionally, the emergence of Virtual Network Operators (VNOs) — which do not own any network infrastructure but lease the required network resources from NOs-offers a cheaper and competitive alternative to network access. As a consequence, NOs are seeking for novel techniques and value added services to avoid being relegated to mere network carriers, and to make a more efficient use of their resources. Furthermore, VNOs require more tunable traffic provisioning methods to reduce the operational costs, and to offer more competitive resource provisioning alternatives to Service Providers (SPs).

At the same time, SPs hold large data centers and clusters to offer different services to end-users, from on-line gaming to multimedia streaming solutions. Many SPs do not own any carrier infrastructure, and thus adopt the overprovisioning imposed by the NOs, and by extension by VNOs, as the option to offer some guaranties in their services. The imposition of such a model is responsible for the limited adoption of Quality of Service (QoS), since overprovisioning is easier to implement and to manage. Opposed to this, the Internet is becoming an interactive and multimedia oriented network, encouraging the development of novel, fully distributed and lightweight quality assessment techniques replacing the legacy (and mostly unused) QoS models. In particular, Quality of Experience (QoE) is gathering the attention from the research community. Jointly with this spreading of multimedia content-which

This work was partially funded by Spanish Ministry of Science and Innovation under contract TEC2009-07041, and the Catalan Government under contract 2009 SGR1508. according to [1] it amounts to a $\sim 27 \%$ of the total Internet traffic and growing - plus the increase in CAPEX and energy consumption caused by network overprovisioning, it is becoming apparent that a new model for network management is required for NOs, for VNOs, and for SPs.

In this paper we propose a promising cross-layer Network Management strategy which allows a SP to dynamically adjust the network resource reservation with its NOs (or VNOs) depending on the end-users perceived QoE of the offered service. Our approach proposes a lightweight mechanism to monitor the QoE at the end-users' applications, using inband signaling with the data center to update the resource reservation with the $\mathrm{NO}$ (or $\mathrm{VNO}$ ) at the network layer accordingly.

We evaluate the performance of our proposal from two different perspectives; first we study the periods with unsatisfied end-users when using our system, compared to the case of having fixed bandwidth allocation. Second, we compare the reduction in operational costs in the SP premises also against the case of fixed bandwidth. We show that even with lower operational costs, the end-user satisfaction is increased.

The rest of the paper is structured as follows. In Section II, we discuss the related work in the area. We continue in Section III with the main contribution of this work, that is, the proposed cross-layer management system. Next, we focus on the simulation driven evaluation of the proposed strategy, highlighting as a use case the performance of our system in a video-streaming scenario. Finally, in Section V, we conclude and outline the open lines in our research.

\section{RELATED WORK}

Network Management (ANM) is an emerging research topic with many open issues [2]. NOs and SPs require autonomic methods to reduce the costs and automating the management of their infrastructure as well as to control and optimize the utilization of resources. In general, such systems are focused on well-known management issues, e.g., resource management [3], QoS/SLA assessment [4], etc.

Our strategy is designed to complement other NMS, offering a promising perspective for dynamic resource reservation. Indeed, we consider cross-layer data by gathering information about the end-users satisfaction-in our approach these data is gathered directly from the end-users' applications without any human intervention-and then performing the required reservation and/or release of resources from the NO (i.e., the 
IP layer and below). This task is typically carried out through QoS mechanisms, requiring highly complex centralized networking infrastructures for managing the data acquisition. To overcome the scalability limitations of these solutions, our approach moves the computational burden of quality assessment to the end-users' applications, which transparently report the system status using in-band signaling.

Since we use the end-users' applications as quality monitors, their location is optimal to measure the user-perceived QoE. QoE stands for the end-user perception of a given service, which can be computed using the Mean Opinion Score (MOS) with techniques such as [5] adapted for video traffic. This paper does not intend to propose new methods of computing the QoE, but rather to exploit existing techniques to provide feedback about the quality offered by the SP, so that the latter can dynamically reserve or release of resources from its NOs to meet its quality policies.

\section{CROSS-LAYER MANAgEMEnT}

In this section, we focus on the description of the main contribution of this paper. In particular, we detail the NMS proposed to perform on-line resource management from the SP towards the NO (vertical signaling) in order to guarantee that the SP's end-users are properly perceiving the offered service (horizontal signaling). As we detail in Fig. 1 the system is composed of the following building blocks:

- Multimedia Service: besides delivering the actual service to end-users, this block also acquires the end-users perceived QoE (horizontal signaling).

- Decision process: it analyzes the perceived QoE reported by the service and decides whether to ask for more resources (vertical signaling), as detailed later in Alg. 1.

- Resource Manager: this block is in charge of the resource management in the data center itself. Since we only focus on the interaction with the NO, in our work we assume that the SP already has mechanisms to monitor and control the data center, along with mechanisms to compute the resources needed by the system to offer the service. Within the Resource Manager we identify:

- Network Resource Manager (NRM): NRM uses the results obtained from the decision process to compute the current service status. New requirements generating specific requests will be sent to the NO providing autonomic capabilities to on-line reservation (or release) of the necessary network resources.

- Billing and accounting: the reservation or release resources is triggered depending on the business model of the SP.

Regarding the Network Operator domain, the blocks interacting with the SP are the Billing and accounting, which is responsible to charge the SP depending on the provisioned resources, and the Network Management System, which enforces the on-line configuration of network devices to comply with the SP requirements. In the following sections, we detail the specific behavior of the QoE assessment within

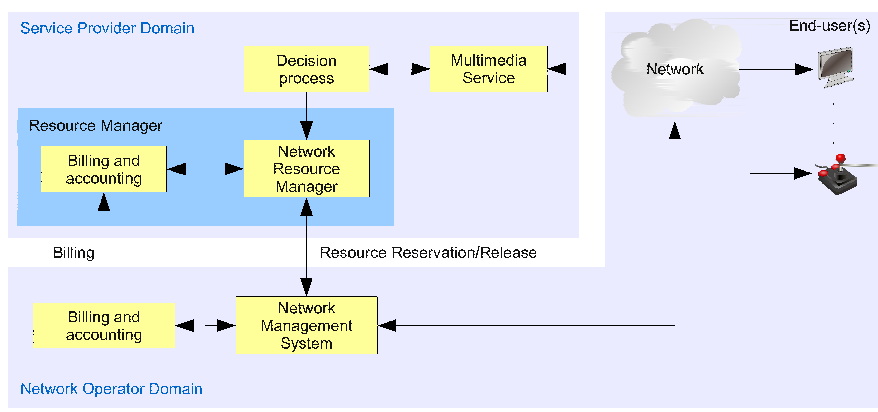

Fig. 1. Building blocks of the Cross-Layer QoE aware NMS.

the Multimedia Service block, the Decision Process, and the Network Resource Manager, excluding the rest of the blocks since they have been studied previously in the literature in works such as [3], [6], and are out of the scope of this paper.

\section{A. Per-service QoE assessment}

Considering the current usage of the Internet, all the services have a potentially high number of end-users, which makes very hard to monitor and analyze in real-time the quality from a centralized location. Despite of this, all such services follow in one way or another the client/server paradigm, which implies that the end-user needs a specific client-side application, namely, a web browser with JavaScript support, or a native application installed to interact with the service. It is thus clear that, these applications offer a vantage point to analyze the delivered service, and since they are running at the end-users' premises, they are good candidates to perform the monitoring. On top of that, using the end-nodes as measurement entities inherently distributes the load of the QoE computation. As a consequence, our approach proposes to upgrade such clientside applications in order to perform the QoE assessment. Using the QoE as a trigger provides a more accurate method for resource reservation than simply using QoS-or available bandwidth-given that it can measure QoE metrics such as response time, or perceived video quality, which cannot be computed with traditional network-based QoS approaches.

For each type of service $(s)$ the SP defines a series of constraints, which are embedded within the client-side application and determine how the QoE is computed. The system then defines a quality threshold $\phi^{(s)} \in[0,1]$ that determines the lower acceptable bound for the service quality; the higher $\phi^{(s)}$ the better is the experienced quality. Even if $\phi$ is service dependent, for video flows, classical thresholds are $\phi=0.7$ (i.e., for MOS values of 3.5). As we shall show in next section, this value is used to manage the network resources in the SP.

The QoE information gathered in the client is sent to the Multimedia Service in the Service Provider Domain and, depending on the service, the frequency of quality notifications can range from a few seconds for real-time services, to tens of seconds for regular services such as Video Streaming, providing a very low rate of updates in the SP. 


\section{B. The decision process}

As discussed above, the decision process is one of the key parts of our infrastructure. In particular, it is in charge of deciding whether the resources used are well suited for the current load depending on the QoE observed by the end-users. In our proposal, the decision process solely uses the perceived QoE as a metric to decide whether to reserve resources, since the decision module has neither knowledge of the current resource reservation nor about bandwidth requirements, such knowledge is in the NRM. This allows a clean design with a generic NRM, while keeping a per service decision process specifically developed to assess the QoE offered.

In a context with thousands of concurrent users, obtaining the perceived QoE of a single end-user is of no practical interest from the SP point of view. On the contrary, having several end-users with degraded quality is relevant, as it might alter the popularity of the service (and of the SP itself).

Hence, we propose the following Assessment strategy, the decision module periodically queries the Service module (cf. Fig. 1) to obtain the perceived QoE for each end-user. The decision process keeps track of the end-users perceived quality in two different ways, depending whether the QoE degradation is localized (i.e., the issues occur within the same regional zone, or within the same network prefix), or global (i.e., there are insufficient reserved resources in the whole system to cope with current end-users' demands). In our initial approach we only consider single domain networks, leaving the study of multi-domain scenarios for future work.

The next step is to decide when a service is considered locally or globally degraded. To this end, we define two different quality thresholds, namely $\rho$ (global) and $\sigma$ (local), as follows:

Definition 1: Let $\rho^{(s)} \in[0,1]$ be the threshold from where a service $S$ is considered globally degraded and more resources are required for the whole SP.

More specifically, $\rho^{(s)}$ is the permitted bound of unsatisfied end-users over the total using the service. Hence, the goal of the system is that, at any instant of time, the ratio of unsatisfied users $v^{(s)}$ is such that $v^{(s)} \leq \rho^{(s)}$, where $v^{(s)}$ is computed using Eq. 1:

$$
v^{(s)}=\frac{\sum_{i=0}^{n-1} \mathcal{U}_{i}}{n}
$$

In Eq. $1, n$ is the amount of end-users of the service, and $\mathcal{U}_{i}$ represents whether the user is unsatisfied, with $\mathcal{U}_{i}$ computed as detailed in Eq. 2:

$$
\mathcal{U}_{i}=\left\{\begin{array}{lll}
1 & , \text { if } q_{i}^{(s)} \leq \phi^{(s)} & \text { (User } i \text { is unsatisfied) } \\
0 & , \text { otherwise } & \text { (User } i \text { is satisfied) }
\end{array}\right.
$$

where $q_{i}$ is the quality experienced by the $i^{\text {th }}$ end-user, and $\phi^{(s)}$ has been previously defined in Section III-A as the lower acceptable bound for the service quality.

Definition 2: Let $\sigma^{(s)} \in[0,1]$ be the threshold from where a service is considered degraded within a network prefix, and more resources are required towards that specific network.

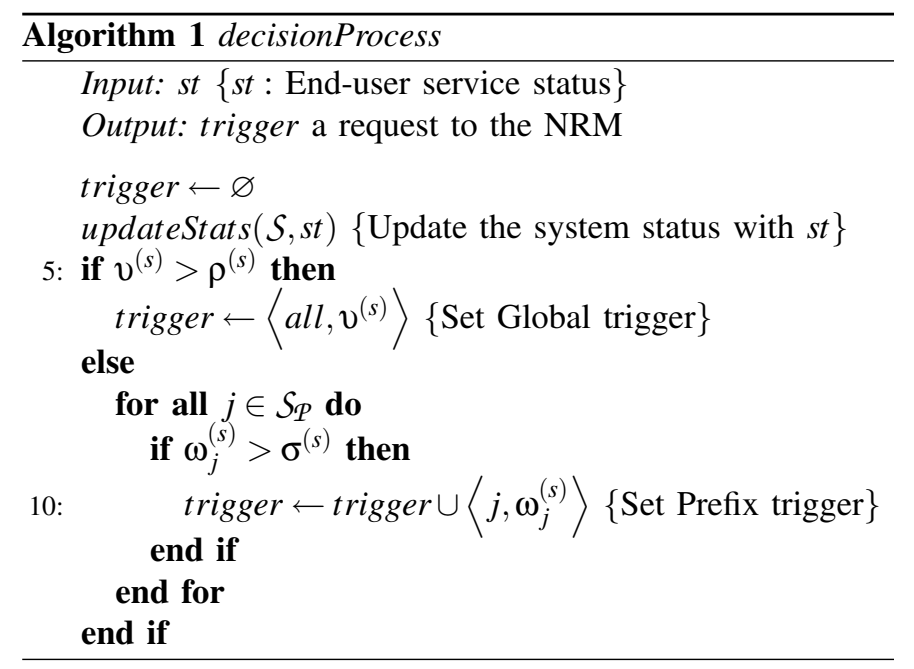

For localized degradations, the goal is that for any network prefix $j \in \mathrm{NO}$, the ratio of unsatisfied users $\omega_{j}^{(s)}$, over those using the service from the local prefix $j$, is such that $\omega_{j}^{(s)} \leq$ $\sigma^{(s)}$. Let's denote $\mathcal{P}=\left\{P_{1}, \ldots, P_{|\mathcal{P}|}\right\}$ as the set of local prefixes using the service. Then, at a given instant of time, the ratio of locally unsatisfied end-users $\omega_{j}^{(s)}$ is computed analogously to $v^{(s)}$ in Eq. 1, but considering only the end-users using prefix $j$. Let $S_{\mathcal{P}}$ be a local status descriptor, containing the ratios of unsatisfied users for each prefix $j \in$ NO. Likewise, let $\mathcal{S}_{\mathscr{P}}^{C}$ be the status descriptor containing the ratios of the overall unsatisfied users. In our system, the ratios of unsatisfied users are stored in a system wide status descriptor $\mathcal{S}$, which holds the status for all the prefixes and users currently in the system: $S=S_{\mathcal{P}} \cup S_{\mathcal{P}}^{C}$.

Algorithm 1 outlines the operations performed by the decision process. The algorithm first updates the overall status $S$ of the data center with the new set of values received from the Service module. After this, the system computes $v^{(s)}$ (Eq. 1), and decides if a global resource request should be sent to the NRM. In case that the number of potentially unsatisfied users is below the threshold $\rho^{(s)}$, the algorithm follows with the assessment of $\omega_{j}^{(s)}$. In this case, the system schedules as many requests as local prefixes under service degradation. As it can be observed, the decision process does not consider the release of resources, which is deferred to the NRM.

\section{Network Resource Manager}

The NRM is in charge of interpreting the values obtained by the decision process, i.e., reserve or release the required resources, and receive feedback about the reservation status from the NO. It might be argued that solely relying on resource usage (e.g., used bandwidth) is enough to provide a proper estimate of the end-users' perception. However, bandwidth usage does not consider events such as lost frames, or enduser wait times for the service, which are critical from the end-user point of view. Additionally, having per end-user QoE information can be used to internally improve the data center behavior, e.g., increasing the priority of the processes serving the unsatisfied end-users or, as we discuss in this work, to 
manage the network resources.

Unlike the decision process, the NRM considers two different aspects regarding the system quality; first the overall resource usage, and second the overall end-user satisfaction. Regarding the resource usage, the system proactively sends a request for more resources towards the $\mathrm{NO}$ before the network reaches an overload situation. To reduce the number of updates, the resources are requested in blocks of fixed size $\delta$, in accordance to the policies imposed by the NO. Regarding the second aspect, whenever the end-user satisfaction drops below the specified bounds $\rho^{(s)}$ or $\sigma^{(s)}$, the NRM will ask for more resources in case the system is close to overloading. As a consequence, the NRM will merge in a single request operation the information obtained from the used network resources (by the Resource Manager) and the satisfaction ratio (from the decision process) with the following criteria:

- If the current system load $L(t)$ is close to the reserved resources $\mathcal{R}(t)$, that is, $L(t) \geq \alpha \mathcal{R}(t)$, where $\alpha \in[0,1)$ is the threshold to avoid network overloading. Then the system reserves $k$ bandwidth blocks complying with $k \delta \geq$ $[1+(1-\alpha)] \mathcal{R}(t)$.

- If the service requires tighter network constraints, i.e., there are still resources but the ratio of unsatisfied endusers is higher than $\rho^{(s)}$ or $\sigma^{(s)}$, the SP must increase the priority of the flows. Such priority increase is usually mapped by the NO to different classes of service, generally classified into four different categories, namely, Gold, Silver, Bronze and Best effort.

Once the requested resources are determined, the system must compute the cost of reserving these resources, and depending on the available budget (or on the reservation policies) the request can be accepted or not. Hence, we propose the following Resource allocation and release policy.

Since the required amount of resources in most services depends on the number of end-users, we use this information to determine the future requirements in terms of resources. The goal of the allocation policy is to minimize the ratio of unsatisfied end-users. As a consequence, the algorithm must quickly react to any potential overloading of resources, while it can have a looser behavior for the release of resources. To this end, we propose two different mechanisms, one suited for Increasing (I) the resource reservation, and the other one for Decreasing (D) it. This behavior is modeled in our system with the expression presented in Eq. 3. Let $r(t)$ denote the amount of required resources at a given instant of time $t$. The policies that we use are based on linear combinations of the history of $r(t)$ as follows:

$$
\begin{aligned}
& L_{I}(t+1)=\Theta_{0} r(t)+\Theta_{1} r(t-1) \\
& L_{D}(t+1)=\Phi_{0} r(t)+\cdots+\Phi_{3} r(t-3)
\end{aligned}
$$

As shown in Eq. 3, for the the increase or release of resources we use a moving average of the last two or four samples of $r(t)$, respectively. The weights in each case are such that: $\sum_{i=0}^{1} \Theta_{i}=1$ and $\sum_{j=0}^{3} \Phi_{j}=1$. Thus, if $L_{I}(t+1) \geq \alpha \mathcal{R}(t)$, then $\mathcal{R}(t+1)=[1+(1-\alpha)] L_{I}(t+1)$, and the system triggers a resource reservation as discussed previously. Otherwise, in the case that $L_{D}(t+1) \leq \alpha \mathcal{R}(t)$, then $\mathcal{R}(t+1)=\mathcal{R}(t)\left[1-\frac{1}{2} L_{D}(t+\right.$ 1)] when $\frac{1}{2} L_{D}(t+1) \mathcal{R}(t)>\delta$, which produces a stepwise smooth decrease of the allocated resources.

\section{Performance Evaluation}

In this section, we evaluate our proposal from two different perspectives. First, we detect the periods with end-user unsatisfaction when using our system, and second, we analyze the reduction in the operational costs observed by the SP. In both cases, we compare our solution against the fixed bandwidth allocation currently offered by NOs.

As a proof of concept, we validate our system by simulating a video streaming service provider with clients requesting a video in real-time. More specifically, the videos have a Constant Bit Rate requirement of $900 \mathrm{Kbps}$, and the users enter and leave the system randomly watching videos with a duration from one minute to one hour. The client applications compute the video quality transparently, using the technique developed in [7], i.e., computing the delivery delays and the packet losses directly from the video frames, and thus assessing the perceived video quality from a single measurement point. This information is sent to the SP, which computes the ratios of satisfaction $v^{(s)}$ and $\omega_{j}^{(s)}$, and decides whether to change the resource reservation. In order to simplify the exposition, we only show here the results for global quality degradations $\left(v^{(s)}\right)$. The simulations of per-prefix degradation lead to similar results.

The simulations compare our approach with the fixed bandwidth allocation present today in SPs. In the set up environment we simulated various flash-crowd events with the following criteria: each simulation lasts around 45 minutes, each flash-crowd event has a duration range spanning from 5 to 10 minutes, e.g., when a specially appealing video is submitted, and there are 5 flash-crowd events per simulation. The rest of the time the average number of users is around one order of magnitude smaller than during the flash-crowd (the overall number of users in the system varies from 1000 to 16000). With these conditions, we run five different simulations changing the number of flash-crowd events that require more resources than the allocated by the system in the case of fixed bandwidth allocation. More specifically, the amount of flash-crowds requiring more resources than the initially allocated ranges from 1 to all 5 . To gather the results, we monitor the allocated bandwidth through reservation requests, the monthly price of the provisioning as detailed in [6], and the periods with service degradation. In particular, we monitor the ratio of service degradation in the system.

Our findings are summarized in Table I, where we show both the case of using our Network Management System (NMS) and using fixed bandwidth (FB). The table first shows the type of flash-crowds, from the trivial case of not having flash-crowds, to the case of having a highly congested site with up to 5 flash-crowds overloading the system during long time periods. The rest of columns detail the percentage of periods with unsatisfied users (column 2), the maximum duration of 


\begin{tabular}{|c|c|c|c|c|c|c|c|c|c|c|}
\hline \multirow{2}{*}{$\begin{array}{l}\text { Flash-Crowds } \\
\text { (FC) }\end{array}$} & \multicolumn{2}{|c|}{ Periods $\mathrm{w} / \mathrm{v}^{(s)}>\rho^{(s)}$} & \multicolumn{2}{|c|}{ Max. Duration (s) } & \multicolumn{2}{|c|}{ Below BW. } & \multicolumn{2}{|c|}{ Avg BW. } & \multicolumn{2}{|c|}{ Monthly Cost (\$) } \\
\hline & NMs & FB & & FB & NMS & FB & NMS & FB & NMS & FB \\
\hline No FC. & $0 \%$ & $0 \%$ & 0 & 0 & $0 \%$ & $0 \%$ & 1425 & 9000 & 11996 & 30000 \\
\hline within limits & $9.9 \%$ & $4.3 \%$ & 35 & 95 & $2.3 \%$ & $0 \%$ & 3953 & 9000 & 16674 & 30000 \\
\hline 1 out-limit & $8.5 \%$ & $13.6 \%$ & 29 & 303 & $2.3 \%$ & $8.8 \%$ & 4584 & 9000 & 19159 & 30000 \\
\hline 2 out-limit & $8.7 \%$ & $23.8 \%$ & 35 & 308 & $2.0 \%$ & $18.1 \%$ & 5536 & 9000 & 22116 & 30000 \\
\hline 3 out-limit & $7.0 \%$ & $36.7 \%$ & 42 & 318 & $2.5 \%$ & $30.1 \%$ & 6791 & 9000 & 25302 & 30000 \\
\hline 4 out-limit & $7.7 \%$ & $39.1 \%$ & 35 & 314 & $2.4 \%$ & $37.8 \%$ & 6727 & 9000 & 26131 & 30000 \\
\hline 5 out-limit & $5.7 \%$ & $49.1 \%$ & 24 & 321 & $1.9 \%$ & $47.1 \%$ & 7918 & 9000 & 29436 & 30000 \\
\hline
\end{tabular}

TABLE I

COMPARISON IN SYSTEM PERFormance With THE PRESENCE OF NETWORK MANAGEMENT System (NMS) AND FiXed BANDWIDTH RESERVATION (FB) FOR THE DIFFERENT OUT-LIMIT FLASH CROWDS (FC).

the user unsatisfaction in seconds (column 3), the percentage of time that the system is underprovisioned-i.e., the user demands are higher than the current provisioning (column 4); the average bandwidth consumption in each simulation (column 5), and the monthly cost in dollars caused by the provisioned bandwidth (column 6).

As it can be observed, our system generally outperforms the fixed bandwidth allocation for the different amount and intensity of flash-crowds. More specially, the amount of time the service is degraded in our system for all the users is independent of the intensity of the flash-crowds, while in the case of having fixed bandwidth it is strongly related, as expected, with the congestion level, in the table it can be noted that the periods with service degradation are always below $10 \%$ for our NMS, raising up to $\sim 50 \%$ for the fixed bandwidth. An aspect worth noticing is that even with flashcrowds within the provisioned limits, there are cases that cause service degradation. The reason for this behavior is that the resource requirements are close to the ones reserved (e.g., link load higher than $80 \%$ ), so the network buffers and the jitter of the video flows derive in mild service degradation, which in some cases cause the $v^{(s)}$ to raise above the threshold $\rho^{(s)}$.

The last analysis we perform in this work refers to the ratio of offered bandwidth against the end-users demands. In Fig. 2-notice the logarithmic scale-we detail the ratio of offered versus demanded bandwidth. In the figure, the value of 1 represents exact balance between offer and demand, values below imply underprovisioning, while values above one refer to an overprovisioned network. As it can be noted, our solution is most of the time above 1 , which means that the resources are slightly overprovisioned by $\delta$ as discussed in the previous section. However, at the edges of the flashcrowd, there is a noticeable underprovisioning caused by the adaptive algorithm. On the other hand, in the case of using fixed bandwidth the system is highly overprovisioned most of the time, however, in the case of severe flash-crowds the existing provisioning is insufficient causing long periods of service degradation, which leads to a poor performance of the service compared to our dynamic reservation solution.

\section{Conclusions And Future Work}

In this paper, we presented a promising proactive approach for Service Providers to efficiently manage traffic provisioning with the Network Operator. The core of the proposed solution uses the end-user perceived QoE of the offered service in order to trigger the resource reservation with the final goal of

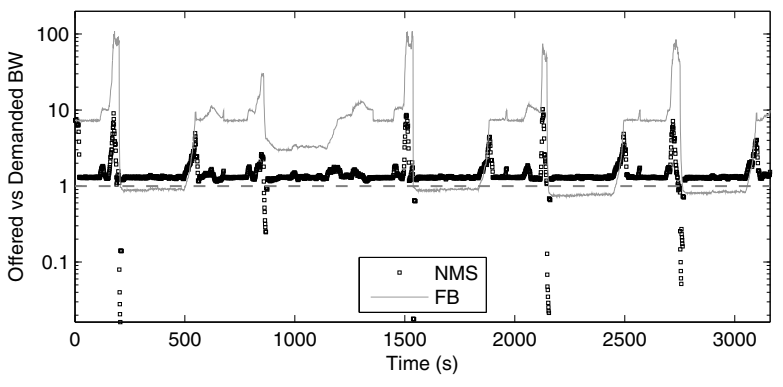

Fig. 2. Offered versus demanded bandwidth for FC. 4 out-limit

minimizing the service degradation periods. The motivation behind the proposal is the increased complexity in terms of cost for NOs to provide overprovisioned networks, and the need of SPs of more efficient and accurate means of resource provisioning, while delivering a reliable service and reducing the operational costs of the network.

To evaluate the performance of our approach we performed a series of simulations with a different set of flash-crowd and high load scenarios that demonstrate that our CrossLayer NMS can, simultaneously, reduce the required network resources and its operational costs, while offering a better service than the classic fixed bandwidth reservation.

As lines open for future work, having a solid business model can raise the interest of Service Providers and Network Operators to implement the system while maintaining a feasible economic model for both parties. Related to this, we plan to extend the platform to multi-domain scenarios.

\section{REFERENCES}

[1] Cisco Systems White Paper. "Cisco Visual Networking Index-Forecast and Methodology, 2007-2012", pages 1-15, June 2008.

[2] Nancy Samaan and Ahmed Karmouch. "Towards autonomic network management: an analysis of current and future research directions". Communications Surveys \& Tutorials, IEEE, 11(3):22-36, Quarter 2009.

[3] Vlad Nae, and et. al. "Efficient management of data center resources for massively multiplayer online games". SC '08: Proceedings of the 2008 ACM/IEEE conference on Supercomputing, pages 1-12, 2008.

[4] René Serral-Gracià, and et. al. "Network performance assessment using adaptive traffic sampling". IFIP Networking, LNCS 4982:252-263, Singapore, May 2008.

[5] Jirka Klaue, and et. al. "EvalVid - A Framework for Video Transmission and Quality Evaluation". In Proceedings of the 13th International Conference on Modelling Techniques and Tools for Computer Performance Evaluation, pages 255-272, 2003.

[6] Hao Wang, and et. al. "Optimal ISP subscription for Internet multihoming: algorithm design and implication analysis". Proceedings IEEE INFOCOM 2005, 2360-2371, March 2005.

[7] René Serral-Gracià and et. al. "Packet Loss based Quality of Experience of multimedia video flows". R. Rep. http://personals.ac.upc.edu/ rserral/research/techreports/psnr_mos.pdf, 2009. 